\title{
PROJECT Method for Optimization of Target Model in DEA
}

\section{Marzieh Moradi Dalini and Abbas Ali Noura}

Department of Mathematics, Kerman Branch, Islamic Azad University, Kerman, Iran

\begin{abstract}
In this paper, we concentrate on performance assessment of special target model in data envelopment analysis (DEA) with use a comprehensive interactive method with name PROJECT method. Interactive methods in multiple objectives programming (MOP) are methods that can help to decision maker (DM) to generate the most preferred solution (MPS). The PROJECT method is able to obtain any efficient solution, contain nonsupport efficient solution and also identify the MPS, even in nonconvex cases. This comprehensive model belongs to the class of interactive local trade-off methods, which is generated by combination the projection of the gradient of an implicit utility function on to the tangent plane of efficient frontier and the reference-point method to search for the best compromise solution within a local region. In this study, we will use the target model since this model focuses on decreasing total input consumption and increasing total output production which results in solving one mathematical model instead of $n$ models, also the PROJECT method to showing the decision maker (DM) preferences in the process of assessing efficiency in the target model in DEA with any prior judgments. Numerical example is examined how the DEA model efficiency analysis can be conducted using the interactive PROJECT for setting realistic target values.
\end{abstract}

Keywords: Multiple objective programming; Interactive PROJECT method; Data envelopment analysis; Utility function; Performance assessment.

\section{Introduction}

In many real decision and planning problems, the problem consists in optimizing (maximizing or minimizing) several conflicting objective functions. In these cases, it is impossible to find a solution which optimized all the objective functions simultaneously. Instead of we can find other types of solutions, called Pareto optimal or no dominated solutions, where none of the objectives can achieve a better value without discounting at least one of the other objective values. Solving (MOP) problem typically means helping a human decision maker (DM) in finding the most preferred solution (MPS) as the final one.

In recent decades, many methods have been developed to solve MOP. They can be classified in four classes according to the role of the DM in the solution process. Interactive methods constitute one of the classes, where in these methods an iterative solution algorithm is formed, its steps are repeated and the DM specifies preference information progressively during the solution process. There are many interactive methods albeit they differ basically from each other in what kind of information is asked from and shown to the DM at each iteration, and in the way the successive solution candidates are calculated. For this reason, interactive methods are powerful tools for solving MOP. Typically, preference information plays an important role in interactive MOP. Examples of types of preference information asked from the DM include the NIMBUS method [1], Wierzbicki's method [2], GRIST method [3] and PROJECT method [4], and many more. Among interactive approaches, we consider two main kind of specifying preference information with names reference points and trade-offs information. The reference points are popular, due to their direct nature. A reference point consists of desirable values for each objective function. Once a reference point is given, an achievement secularizing function is minimized to find a no dominated solution. In practice this means that the reference point is projected to the set of no dominated solutions and any no dominated solution can be found by altering the reference point. When solving a multiobjective problem, by tradeoffs can confidence that the decisions to be made are the right ones for the DM, it important to understand the tradeoffs related to different Pareto optimal solutions. Most of the interactive methods based on local tradeoffs assume convexity conditions in MOP problem, which is too restrictive in many real-life applications, but using a reference point procedure makes it possible to generation any efficient solutions, even if the location be nonconvex.

Furthermore, relationships between reference point techniques and local tradeoffs are analyzed [4]. In this paper, we concentrate on interactive PROJECT method [4], when this model belongs to the class of interactive local tradeoff methods with name gradient-based interactive step tradeoff (GRIST) method [3] and a local search way that inherits the benefits of the reference point method to search for the best compromise solution. Therefor with uses the PROJECT model we can obtain all efficient solutions when to exist in both convex and nonconvex locals. In this model the DMs preferences can be better reflected in a solution generated by using suitable weights in the achievement secularizing function, which leads to the generation of solutions which can maintain the local tradeoffs.

On the other hand, data envelopment analysis (DEA) and multiple objective linear programming (MOLP) can be used as tools in management control and planning. The structures of theses two of models have much in common, but DEA is directed to estimating past performances as part of the management control function and MOLP to planning future performance [5], the relation between DEA and MOLP have been discussed from several standpoints by many authors. For instance, Golany first suggested an interactive model combining both DEA and MOLP methods where the DM is assumed to be able to allocate a set of point levels as resources and to select the most preferred set of output levels from a set of alternative points on the efficient frontier [6].

${ }^{*}$ Corresponding author: Abbas Ali Noura, Department of Mathematics, Kerman Branch, Islamic Azad University, Kerman, Iran, Tel: 50-33210043-034; E-mail: marziehmoradi99@yahoo.com

Received October 21, 2016; Accepted September 18, 2017; Published September 30, 2017

Citation: Dalini MM, Noura AA (2017) PROJECT Method for Optimization of Target Model in DEA. Global J Technol Optim 8: 219. doi: 10.4172/2229-8711.1000219

Copyright: $\odot 2017$ Dalini MM, et al. This is an open-access article distributed under the terms of the Creative Commons Attribution License, which permits unrestricted use, distribution, and reproduction in any medium, provided the original author and source are credited. 
Hosseinzadeh Lotfi et al. obtained an equivalence relationship between min-ordering formulation in MOLP and DEA based on output-oriented CCR dual model [7]. Also Yang et al. established the equivalence relationship between the output-oriented DEA dual models and minimal reference point approach of MOLP, then they showing how a DEA problem can be solved interactively without any prior judgment by transforming it into a MOLP formulation [8]. Following than, Malekmohammadi and Hosienzadeh Lotfi improved equivalence between target setting model in DEA (this model considered both the decreases in total input consumption and the increase in total output production) and minimal reference point approach of MOLP, at last they solved interactively the target model by the GRIST method [9]. According to the GRIST method defined on based just convex regions, in this paper, we will use PROJECT method, it must be pointed out that with use the PROJECT method we can obtain any efficient solutions (contain nonsupport efficient solution) and also identify the MPS, even in nonconvex cases.

This paper is organized as follows. In section 2, we set up notation and terminology, including the GRIST and PROJECT methods introduced; also we introduce the target model in DEA. Section 3, we will investigation performance assessment in the target model with using PROJECT method. Section 4 contains a numerical example, and the conclusion is provided in section 5 .

\section{Preliminaries}

\section{Notation and terminology}

Let us consider multi objective optimization problems of the form

$$
\begin{aligned}
& \max \left[f_{1}(\lambda), \ldots, f_{k}(\lambda), \ldots, f_{s}(\lambda)\right] \\
& \text { s.t } \quad \lambda \in \Omega \\
& \text { where } \\
& \Omega=\left\{\lambda \mid g_{j}(\lambda) \leq 0, \quad h_{l}(\lambda)=0 ; \quad j=1, \ldots, k_{1} \quad l=1, \ldots, k_{2}\right\} \subset R^{n}
\end{aligned}
$$

Where $\lambda=\left(\lambda_{1} \ldots \lambda_{n}\right)^{T}$ is an $\mathrm{n}$-dimensional vector of decision variables, $\Omega$ is the feasible region, $Z=f(\Omega)$ is the criterion space, and $z=f(\lambda)$ is a criterion vector where $z \in Z$ if $\lambda \in \Omega$ exists. Suppose that $f_{k}(\lambda)$ are differentiable objective functions, and $g_{j}(\lambda), h_{l}(\lambda)$ are differentiable constraint functions, $\Omega$ is compact, and its interior is nonempty.

In MOP, there is generally no feasible solution that can maximize all concept of efficient solution. A decision vector $\lambda^{*} \in \Omega$ is called efficient or Pareto optimal for (1) if there does not exist another $\lambda \in \Omega$ such as $f_{k}\left(\lambda^{*}\right) \leq f_{k}(\lambda) \quad \forall k=1, \ldots, s$ and $f_{\tau}\left(\lambda^{*}\right)<f_{\tau}(\lambda)$ for at least one index $\tau$.

It is useful to know the bounds for the objective vectors in the nondominated objective set, since the set of nondominated objective vector contain more than one vector. Specifically, In MOP ideal and nadir values are defined by

$$
\begin{aligned}
& z_{k}^{*}=\max _{\lambda \in E} f_{k}(\lambda)=\max _{\lambda \in \Omega} f_{k}(\lambda) \quad \forall k=1 \ldots S \quad z^{*}=\left(z_{1}^{*}, \ldots, z_{k}^{*}\right)^{T} \\
& m_{k}^{*}=\min _{\lambda \in E} f_{k}(\lambda) \quad \forall k=1 \ldots S \quad m^{*}=\left(m_{1}^{*}, \ldots, m_{k}^{*}\right)^{T}
\end{aligned}
$$

Ideal values are obtained easily by means of maximizing each objective function separately. In contrast, nadir values are not easy to obtain and, when estimated from the pay-off table, the values achieved are not necessarily good approximations. Both the ideal vector and the nadir vector are used frequently to normalize the objective functions. Let $\mathrm{u}$ be the DM's global utility function

$$
u \equiv u\left(f_{1}(\lambda), \ldots, f_{k}(\lambda), \ldots, f_{s}(\lambda)\right.
$$

And assume $u$ is a continuously differentiable and strictly increasing nonlinear function, defined as follows $\partial u$

$$
\frac{\partial u}{\partial f_{k}}>0 \quad k=1, \ldots, s
$$

An efficient solution that maximizes the utility function $u$, also known as the MPS, is found by solving problem (1) with u maximized. When $\mathrm{u}$ cannot be expressed explicitly, the MPS can be searched interactively with $\mathrm{u}$ progressively assessed using the DM's local preferences. If $\mathrm{u}$ can be expressed explicitly, the MPS can be reached by solving the following problem:

$$
\begin{array}{ll}
\max & u\left(f_{1}(\lambda), \ldots, f_{k}(\lambda), \ldots, f_{s}(\lambda)\right) \\
\text { st } & \lambda \in \Omega
\end{array}
$$

The aim of an interactive method in MOP is to improve DM's utility in an iterative way, especially if the utility function is not expressed explicitly. Wierzbicki (1980) proposed to use the achievement function to generate a solution according to the DM's local preferences. In this case, consider the following achievement function.

$$
s\left(q^{t}, f(\lambda) \mu^{t}\right)=\max _{k=1 \ldots s}\left\{\mu_{k}^{t}\left(q_{k}^{t}-f_{k}(\lambda)\right\}\right.
$$

Where $\mathrm{t}$ designate the iteration number, $q^{t}=\left(q_{1}^{t}, \ldots, q_{s}^{t}\right)$ is the reference point (target values) provided by DM, and $\mu^{t}=\left(\mu_{1}^{t}, \ldots, \mu_{s}^{t}\right)$ are achievement function weights and $\mu_{k}^{t}>0$. Minimizing this function

$$
\min _{\lambda \in \Omega}=s\left(q^{t}, f(\lambda) \mu^{t}\right)
$$

A new solution is obtained, denoted by $\lambda^{t+1}$ corresponding to the $(t+1)$ th iteration. Let $f^{t+1}=f\left(\lambda^{t+1}\right)$, and equivalently (5) becomes

$$
\begin{array}{ll}
\min _{\lambda, \alpha} & \alpha \\
\text { s.t } & \mu_{k}^{t}\left(q_{k}^{t}-f_{k}(\lambda) \leq \alpha \quad k=1 \ldots s\right. \\
& \lambda \in \Omega
\end{array}
$$

\section{GRIST algorithm}

The Gradient-based interactive step tradeoff (GRIST) method can be used to identify a normal vector at a given efficient solution on the efficient frontier and to design an interactive procedure for searching for the MPS that maximize the DM's implicit utility function. We first describe the method, and then develop the tradeoff approach. Suppose for a given positive weight vector $w^{t}=\left\{w_{1}^{t}, \ldots, w_{k}^{t}, \ldots, w_{s}^{t}\right\}$ the optimal solution $\lambda^{t}=\left\{\lambda_{1}^{t}, \ldots, \lambda_{k}^{t}, \ldots, \lambda_{s}^{t}\right\}$ generated by solving the following minimal problem

$$
\begin{aligned}
& \min \quad \alpha \\
& \begin{array}{l}
\text { s.t } \quad w_{k}\left(z_{k}^{*}-f_{k}(\lambda) \leq \alpha\right. \\
\quad \lambda \in \Omega
\end{array} \\
& \text { Where } w_{k}^{t}=\frac{1}{z_{k}^{*}-f_{k}^{t}} \quad \forall k=1, \ldots, s, s
\end{aligned}
$$

Suppose that $\beta_{k}^{t} \quad \forall k=1, \ldots, s$ are the optimal Lagrange multipliers associated with the constraints

$w_{k}\left(z_{k}^{*}-f_{k}(\lambda) \leq \alpha \quad \forall k=1, \ldots, s\right.$. It is proved that the normal vector $N^{t} f\left(\lambda^{k}\right)$

on the efficient frontier is given by

$$
N^{t}=\left[w_{1}^{t} \beta_{1}^{t}, \ldots, w_{k}^{t} \beta_{k}^{t}, \ldots, w_{s}^{t} \beta_{s}^{t}\right]^{T}
$$

Suppose the implicit utility function of the DM is denoted by $u\left(f(\lambda)\right.$ at the solution $f\left(\lambda^{t}\right)$ is given by 


$$
\nabla u\left(\lambda^{t}\right)=\left[\frac{\partial u}{\partial f_{1}}, \ldots, \frac{\partial u}{\partial f_{s}}\right]^{T}
$$

Because the utility function is not known explicitly, a utility gradient needs to be estimated using DM's indifference tradeoffs or marginal rate of substitution as follows. Without loss of generally, set the first objective as the reference objective. Then the indifference tradeoff $M^{t}$ between the first and the kth objectives and the marginal rate of substation $M^{t}$ at $f\left(\lambda^{t}\right)$ are define by

$$
M_{1 k}^{t}=-\frac{d f_{1}^{t}}{d f_{k}^{t}} \quad \text { and } \quad M^{t}=\left[1, M_{12}^{t}, \ldots, M_{1 k}^{t}, \ldots, M_{1 s}^{t}\right]^{T}
$$

Which provides an approximation of the normalized utility gradient and denoted by

$$
\begin{aligned}
& M^{t}=\overline{\nabla u}\left(f^{t}\right) \\
& =\frac{\nabla u\left(f^{t}\right)}{\partial u / \partial f_{k}}=\frac{\left(\frac{\partial u}{\partial f_{1}}, \ldots, \frac{\partial u}{\partial f_{k}}\right)}{\partial u / \partial f_{k}} \cong\left(m_{12}^{t}, \ldots, m_{1 s}^{t}\right)^{T}
\end{aligned}
$$

Considering at the MPS, the following optimal indifference tradeoff between $f_{1}(\lambda)$ and kth objective can be calculated using the following equation

$$
d f_{k}^{t}=-d f_{1}^{t} \frac{w_{1}^{t} \beta_{1}^{t}}{w_{k}^{t} \beta_{k}^{t}}
$$

The projection $\nabla u\left(f^{t}\right)$ onto the tangent hyper plane of the efficient set is an ascending direction in DM's utility, which denoted by $\Delta F^{t}=\left[\Delta f_{1}^{t}, \Delta f_{2}^{t}, \ldots, \Delta f_{s}^{t}\right]^{T}$

$$
\begin{aligned}
& \Delta u^{t}=M^{t}-c \cdot N^{t} \\
& c=\frac{\left[\left(M^{t}\right)^{T} N^{t}\right]}{\left[\left(N^{t}\right)^{T} N^{t}\right]}=\frac{\sum_{r=1}^{s} m_{k} w_{k}^{t} \cdot \beta_{k}^{t}}{\sum_{r=1}^{s}\left(w_{k}^{t} \cdot \beta_{k}^{t}\right)^{2}}
\end{aligned}
$$

The current solution $\lambda^{t}$ is the MPS if $\Delta F^{t}=0$. Note that in this case $\nabla u\left(f^{t}\right)$ and $N^{t}$ are proportional vectors. If $\lambda^{t}$ is not an MPS, $\Delta F^{t} \neq 0$ evidently, if $\lambda^{t}$ is an efficient solution, to improve an objective, it is necessary that some of the other objective are victim.

\section{Project method}

The PROJECT method was proposed in based on the GRIST method and together with the methodology of reference point techniques [10]. The GRIST method can be used to identify a normal vector at a given efficient solution on the efficient frontier. The PROJECT method, offers a better way to search for a new solution that the GRIST method as local tradeoffs provided by the DM are maintained through the use of reference point approaches.

Steps of the Algorithm:

The ideal values $z^{*}=\left(z_{1}^{*}, \ldots, z_{s}^{*}\right)^{T}$ and nadir values $m^{*}=\left(m_{1}^{*}, \ldots, m_{s}^{*}\right)^{T}$ are calculated.

Iteration $t=0$. Select an initial point $q_{k}^{i}=z_{i}$. An initial solution can be obtained by solving problem (6) by setting $q_{k}^{t}=z_{k}^{*}$ and $\mu_{r}^{t}=\left(\frac{1}{z_{k}^{*}-m_{k}^{*}}\right) \quad \forall k=1, \ldots, s$.

Calculate the normal $N^{t}$ at $\lambda^{t}$ using (8).

The DM is asked to obtain local indifference tradeoffs on objectives, so that the vector of the normalized gradient can be defined.

$$
M^{t}=\overline{\nabla u}\left(f^{t}\right)=\left(m_{1}^{t}, \ldots, m_{s}^{t}\right)^{T}
$$

Calculate the projection $\Delta F^{t}=\left[\Delta f_{1}^{t}, \Delta f_{2}^{t}, \ldots, \Delta f_{s}^{t}\right]^{T}$ of $M^{t}$ onto the tangent hyper plane of the efficient set at, $\lambda^{t}$ using (9). Given

$$
\begin{aligned}
& J_{1}=\left\{k \mid \Delta f_{k}^{t}>0, \quad k \in\{1 \ldots s\}\right\} \\
& J_{2}=\left\{k \mid d f_{k}^{t}=0, \quad k \in\{1 \ldots s\}\right\}
\end{aligned}
$$

If $\Delta F^{t}=0$, then $\left(\lambda^{t}, f^{t}\right)$ is the MPS, and the iteration is stopped.

If $\Delta F^{t} \neq 0$ and $\Delta f_{k}^{t}=0$ for $k \in J_{2}$, the perturbation technique (11) is used, and then continue.

$$
\begin{aligned}
& \Delta f_{j}^{t}=-\mu \cdot\left|f_{j}^{t}\right| \\
& 0 \leq \mu \leq \frac{\sum_{r \in J_{1}} m_{k}^{t} \cdot \nabla f_{k}^{t}}{\sum_{j \in J_{2}} m_{j}^{t} \cdot\left|f_{j}^{t}\right|}
\end{aligned}
$$

5) Calculate the desired step size could be determined by

$$
\begin{aligned}
& s_{k}^{t}=\frac{z_{k}^{*}-f_{k}^{t}}{\left|\Delta f_{k}^{t}\right|} \quad \forall k \in J_{1}=\left\{k \mid \Delta f_{k}^{t}>0, k \in\{1 \ldots s\}\right\} \\
& s_{k}^{t}=\frac{f_{k}^{t}-m_{k}^{t}}{\left|\Delta f_{k}^{t}\right|} \quad \forall k \in J_{2}=\left\{k \mid \Delta f_{k}^{t}<0, k \in\{1 \ldots s\}\right\} \\
& s_{\max }^{t}=\min _{1 \leq k \leq s} s_{k}^{t}
\end{aligned}
$$

The step size $S^{t}$ is taking interval $\left[0, s_{\max }^{t}\right]$ and given that $s_{l}^{t}=\frac{l}{C_{s}} \cdot s_{\max }^{t} \quad l=1,2 \ldots, C_{S}$

Where Cs is an integer which could be regulated to change accuracy of, then, the following vectors of value on the objectives are shown to the DM:

$\left(f_{1}^{t}+s_{1}^{t} \cdot \Delta f_{1}^{t}, \ldots, f_{s}^{t}+s_{l}^{t} . \Delta f_{s}^{t}\right) \quad l=1 \ldots C_{s}$

The new reference point is defined by $q^{t}=\left(q_{1}^{t}, \ldots, q_{s}^{t}\right)$

Where $q_{k}^{t}=f_{k}^{t}+s^{t} . \Delta f_{k}^{t} \quad \forall k=1 \ldots s$

Solve problem (6), where $\mu_{k}^{t}=\frac{1}{\left|q_{k}^{t}-f_{k}^{t}\right|} \quad \forall k=1 \ldots s$. Let
$\left.f^{*}\right)$ be a solution.

Show the solution $\left(\lambda^{*}, f^{*}\right)$ to the DM. If $u\left(f^{*}\right) \geq u\left(f^{t}\right)$, then let, then let $\lambda^{t+1}=\lambda^{*}$ and $f^{t+1}=f^{*}$. Set $t=t+1$ and go to step 2 .

Otherwise, let

$s^{t}:=s^{t} \cdot \min \left\{\left|1-\frac{\left\|q^{t}-f^{*}\right\|_{2}}{\left\|q^{t}-f^{t}\right\|_{2}}\right|, \frac{1}{2}\right\}$

And go to step 6

\section{Target setting and data envelopment analysis}

Data envelopment analysis (DEA) is a mathematical programming model for calculating relative efficiency of decision making units. The first DEA was suggested by Charnes et al., which is a nonlinear fractional mathematical programming model, known as the CCR model [11] 
The original DEA models do not include DM's preferences structure or value judgments in measuring relative efficiency and setting target values, so there is minimal input from DM. Allen et al. defined value judgment as "logical constructs, incorporated within an efficiency assessment study, reflecting the DM's preferences in the process of assessing efficiency" [12]. By incorporating the DM's value judgments, it showed how a DEA problem can be solved interactively without any prior judgment by transformed it into a MOLP formulation [13]. One of the important issues in DEA is evaluating the efficiency of the DMUs. Since it is important to known whether or not the DMU projected on to the efficient frontier is acceptable and ideal for the decision makers, many researches have been carried out under the names of target setting and allocating resource.

Suppose an organization has $\mathrm{n}$ decision-making units (DMUs) while $(j, r=1, \ldots, n)$, producer s outputs denoted by $y_{k r}$ (the kth output of unit $\mathrm{r}$ ), for $k=1, \ldots, s$ and consumes $m$ inputs denoted by $x_{\dot{g}}$ (the ith input of unit j) for $i=1, \ldots, m$. Also $p_{i}^{-}, p_{k}^{+}$the user specified constant reflecting the DM's preferences over the improvement of input/output components, $\theta_{i}, Z_{k}$ the contraction rate of input $\mathrm{i}$ and expansion of output $\mathrm{r}$, respectively, and $g_{i}, g_{k}^{\prime}$, the bounds for total estimated targets of input $\mathrm{i}$ and output $\mathrm{k}$. The vector $\left(\lambda_{1 r}, \lambda_{2 r}, \ldots, \lambda_{n}\right)$, such that $\sum_{i=1}^{n} \lambda_{r} \forall r$, is imposed for convex combination of inputs or outputs for $n$ DMUs.

Maximum muscle force via the following mathematical function. The muscle forces (left side/right side) were as follows: masseter (176.86 $\mathrm{N} / 161.32 \mathrm{~N})$, temporalis $(104.71 \mathrm{~N} / 125.80 \mathrm{~N})$, and medial pterygoid $(87.69 \mathrm{~N} / 79.18 \mathrm{~N})$.

Young's modulus of the SLAffinity layer was $43.65 \mathrm{GPa}$, determined using a TriboLab Nano indenter (Hysitron) with a diamond indenter (tip radius: $150 \mathrm{~nm}$ ). In the present study, stress distributions of SLAffinity-treated dental implants with (SLAffinity-Ti-SB) and without SB cell therapy (SLAffinity-Ti) were compared [14].

$$
\begin{array}{ll}
\underset{\lambda_{j}, Z_{k}, \theta_{i}}{\operatorname{Max}} & \sum_{k \in O} P_{k}^{+} Z_{k}-\sum_{i \in I} P_{i}^{-} \theta_{i} \\
\$ & \sum_{r=1}^{n} \sum_{j=1}^{n} \lambda_{j} x_{j}=\theta_{i} \sum_{j=1}^{n} x_{j} \quad i \in I \\
& \sum_{r=1}^{n} \sum_{j=1}^{n} \lambda_{j} y_{k}=Z_{k} \sum_{r=1}^{n} y_{k} \quad k \in O \\
& \sum_{j=1}^{n} \theta_{i} x_{j} \geq g_{i} \quad i \in I_{f} \\
& \sum_{r=1}^{n} Z_{k} y_{k} \leq g_{k}^{\prime} \quad k \in O_{f} \\
& \sum_{j=1}^{n} \lambda_{j}=1 \quad \forall r \\
& \lambda_{j} \geq 0 \quad \forall j, r
\end{array}
$$

\section{An Interactive Procedure to Find MPSs for Management Planning and Target Setting}

Yang et al. (2009) established the equivalence relationship between the output-oriented DEA dual models and minimal reference point approach of MOLP, Following that, Hossinzadeh Lotfi et al. (2011), proposed the target model which computes one rather than $n$ mathematical programming problems and reduce total input and increases total output simultaneously. Additionally they investigated the equivalence between this model and minimal reference point formulation.

\section{Connection between the target setting in DEA and MOLP model}

The target model, as shown in formulation (15), can be equivalently re-written as follows:

$$
\begin{array}{ll}
\operatorname{Max}_{\lambda_{j}, Z_{k}, \theta_{i}} & \sum_{k \in O} P_{k}^{+} Z_{k}-\sum_{i \in I} P_{i}^{-} \theta_{i} \\
\text { st } \quad & \sum_{r=1}^{n} \sum_{j=1}^{n} \lambda_{j r} x_{i j}=\theta_{i} \sum_{j=1}^{n} x_{i j} \quad i \in I \\
& \sum_{r=1}^{n} \sum_{j=1}^{n} \lambda_{j r} y_{k j}=Z_{k} \sum_{r=1}^{n} y_{k r} \quad k \in O \\
& \sum_{j=1}^{n} \theta_{i} x_{i j} \geq g_{i} \quad i \in I_{f} \\
& \sum_{r=1}^{n} Z_{k} y_{k r} \leq g_{k}^{\prime} \quad k \in O_{f} \quad \\
& \lambda \in \Omega=\left\{\lambda \mid \sum_{j=1}^{n} \lambda_{j r}=1 \quad \forall r, \lambda_{j r} \geq 0 \quad \forall r \forall j\right\}
\end{array}
$$

In this subsection, we obtain an equivalent for formulation (16), so, we conduct efficiency analysis, and interactive MOLP approach will be used to locate the MPS or set target values for all DMUs simultaneously. Assume in model (16) the kth total composite output and ith total composite input are renamed by $\bar{f}_{k}(\lambda), \widetilde{f}_{i}(\lambda)$ respectively.

$$
\begin{aligned}
& \bar{f}_{k}(\lambda)=\sum_{r=1}^{n} \sum_{j=1}^{n} \lambda_{j} y_{k} \quad \widetilde{f}_{i}(\lambda)=\sum_{r=1}^{n} \sum_{j=1}^{n} \lambda_{j} x_{j}, k=1 \ldots s \quad i=1 . m, \\
& \lambda=\left(\lambda_{11}, \lambda_{j r}, \ldots, \lambda_{n n}\right)^{T}
\end{aligned}
$$

Suppose that the maximum feasible value of the kth total composite output and the minimum feasible value for the ith total composite input are renamed by $f_{k}^{\prime}=\bar{f}_{k}\left(\lambda^{*}\right), f_{i}^{\prime \prime}=\widetilde{f}_{i}\left(\lambda^{*}\right)$, respectively, where $\lambda^{*}$ can be computed by solving the following optimization problems:

$$
\begin{array}{ll}
\text { Max } & \sum_{r=1}^{n} \sum_{j=1}^{n} \lambda_{j r} y_{k} \\
\text { s.t } \quad & \lambda \in \Omega \\
\text { Min } & \sum_{r=1}^{n} \sum_{j=1}^{n} \lambda_{j r} x_{j} \\
\text { s.t } \quad & \lambda \in \Omega
\end{array}
$$

Note that $f^{\prime}=\left[f_{1}^{\prime}, \ldots, f_{s}^{\prime}\right]$ and $f^{\prime \prime}=\left[f_{1}^{\prime \prime}, \ldots, f_{m}^{\prime \prime}\right]$ are ideal points in the objective space spanned by (18) for $\lambda \in \Omega$. The equivalent technic can established as follows:

$$
\begin{aligned}
& \text { Suppose } \sum_{j=1}^{n} x_{\ddot{j}}>0 \quad i=1, \ldots, m \text { and }, \sum_{r=1}^{n} y_{k r}>0 \quad k=1, . ., s \\
& w_{k}=\frac{1}{\sum_{r=1}^{n} y_{k}} \quad \forall k, v_{i}=\frac{1}{\sum_{j=1}^{n} x_{j}} \quad \forall i
\end{aligned}
$$

Where $w_{k}, v_{i}$ are used for changing the interactive process of

locating the MPS

$$
\bar{f}_{k}^{*}=\frac{F^{\max }}{w_{k}}=F^{\max } \sum_{r=1}^{n} y_{k}, \quad \tilde{f}_{i}^{*}=\frac{H^{\min }}{v_{i}}=H^{\min } \sum_{j=1}^{n} x_{j}
$$


$\tau_{i}=\theta_{i}-H^{\min }, \quad \gamma_{k}=F^{\max }-Z_{k}$

With

$$
\begin{aligned}
& F^{\mathrm{max}}=\max _{1 \leq k \leq s}\left\{w_{k} f_{k}^{\prime}\right\}=\max _{1 \leq k \leq s}\left\{\frac{f_{k}^{\prime}}{\sum_{r=1}^{n} y_{k r}}\right\} \\
& H^{\min }=\min _{1 \leq i \leq m}\left\{v_{i} f_{i}^{\prime \prime}\right\}=\min _{1 \leq i \leq m}\left\{\frac{f_{i}^{\prime \prime}}{\sum_{j=1}^{n} x_{j}}\right\}
\end{aligned}
$$

The equivalent method can be presented as follows:

$$
\begin{array}{ll}
\operatorname{Max}_{\lambda_{j}, Z_{k}, \theta_{i}} & \sum_{k \in O} P_{k}^{+} Z_{k}-\sum_{i \in I} P_{i}^{-} \theta_{i} \\
\text { st } & \tilde{f}_{i}(\lambda)-\theta_{i} \frac{1}{v_{i}}=0 \quad i=1, \ldots, m \\
& Z_{k} \frac{1}{w_{k}}-\bar{f}_{k}(\lambda)=0 \quad k=1, \ldots, s \\
& g_{i} v_{i} \leq \theta_{i} \quad i \in I_{f} \\
& g_{k}^{\prime} w_{k} \geq Z_{k} \quad k \in O_{f}, \quad \lambda \in \Omega
\end{array}
$$

The first $\mathrm{m}$ objective constraint in (23) can be equivalently as follows, where ….. means "is equivalent". For any, we have:

$$
\begin{aligned}
& Z_{k} \frac{1}{w_{k}} \bar{f}_{k}(\lambda)=0 \Leftrightarrow-w_{k} \bar{f}_{k}(\lambda)=-Z_{k} \Leftrightarrow F^{\max }- \\
& w_{k} \bar{f}_{k}(\lambda)=F^{\max }-Z_{k} \Leftrightarrow w_{k}\left(\bar{f}_{k}^{*}-\bar{f}_{k}(\lambda)=F^{\max }-Z_{k}\right.
\end{aligned}
$$

Assume that $P_{k}^{+}=P_{i}^{-}=1, k \in O, i \in I$. The objective function model (23) becomes

$$
\begin{aligned}
& \underset{\lambda_{j}, Z_{k}, \theta_{i}}{\operatorname{Max}}\left(\sum_{k \in O} Z_{k}-\sum_{i \in I} \theta_{i}\right)=\underset{\lambda_{j} Z_{k}, \theta_{i}}{\operatorname{Min}}\left(\sum_{i \in I} \theta_{i}+\sum_{k \in O}\left(-Z_{k}\right)\right) \Rightarrow \\
& \operatorname{Min}\left(\sum_{i \in I}\left(\theta_{i}-H^{\mathrm{min}}\right)+\sum_{k \in O}\left(F^{\max }-Z_{k}\right)=\operatorname{Min}\left(\sum_{i \in I} \tau_{i}+\sum_{k \in O} \gamma_{k}\right)\right.
\end{aligned}
$$

Note that for any $\lambda \in \Omega$

$$
\begin{aligned}
& F^{\max }-Z_{k} \geq w_{k} \bar{f}_{k}^{*}-Z_{k} \geq w_{k} \bar{f}_{k}(\lambda)-Z_{k}=0 \quad k=1, \ldots, s \\
& \theta_{i}-H^{\min } \geq \theta_{i}-v_{i} \widetilde{f}_{i}^{*} \geq \theta_{i}-v_{i}\left(\widetilde{f}_{i}(\lambda)=0 \quad i=1, \ldots, m\right. \\
& \bar{f}_{k}^{*}=\frac{F^{\max }}{w_{k}} \geq \frac{w_{k} f_{k}^{\prime}}{w_{k}}=f_{k}^{\prime}=\max _{\lambda \in \Omega} \bar{f}_{k}(\lambda) \quad k=1, \ldots, s \\
& f_{i}^{*}=\frac{H^{\min }}{v_{i}} \leq \frac{v_{i} f_{i}^{\prime \prime}}{v_{i}}=f_{i}^{\prime \prime}=\min _{\lambda \in \Omega} \widetilde{f}_{i}(\lambda) \quad i=1, \ldots, m
\end{aligned}
$$

The above analysis display that if the reference point in model (17) is set by

$$
\bar{f}^{*}=\left[\bar{f}_{1}^{*}, \ldots, \bar{f}_{s}^{*}\right]^{T}=F^{\max }\left[\sum_{r=1}^{n} y_{1 r}, \ldots, \sum_{r=1}^{n} y_{s}\right]^{T}
$$

$\widetilde{f}^{*}=\left[\widetilde{f}_{1}^{*}, \ldots, \widetilde{f}_{s}^{*}\right]^{T}=H^{\min }\left[\sum_{j=1}^{n} x_{1 j}, \ldots, \sum_{j=1}^{n} y_{\dot{m}}\right]^{T}$ are defined by (19), and $g_{i} v_{i}-H^{\min } \leq \theta_{i}-H^{\min }=\tau_{i}, \quad g_{k}^{\prime} w_{k} \geq Z_{k}-F^{\max }=\gamma_{k}$ then model (15) will be same to the following minimal reference point formulation

$$
\begin{array}{cl}
\text { Min } & \sum_{i \in I} \tau_{i}-\sum_{k \in O} \gamma_{k} \\
\text { st } \quad & v_{i}\left(\tilde{f}_{i}(\lambda)-\tilde{f}_{i}^{*}\right)=\tau_{i} \quad i=I \\
& w_{k}\left(\bar{f}_{k}^{*}-\bar{f}_{k}(\lambda)\right)=\gamma_{k} \quad k=O \\
& g_{i} v_{i}-H^{\min } \leq \tau_{i} \quad i \in I_{f} \\
& g_{k}^{\prime} w_{k}-M \leq \gamma_{k} \quad k \in O_{f} \\
\sum_{j=1}^{n} \lambda_{j r}=1, \quad & \forall r \quad \lambda_{j r} \geq 0, \quad M \geq 0 \quad j, r=1, \ldots, n
\end{array}
$$

\section{Interactive PROJRCT method for identify any efficient solu- tion}

In this subsection we will suggest interactive PROJECT method for solve the target model and search the MPS on the efficient frontier. It would be worth mentioning that with use the PROJECT method we can obtain any efficient solutions (contain nonsupport efficient solution) and also identify the MPS, even in nonconvex cases. The PROJECT method, however, offers a better way to search for a new solution than the GRIST method as local tradeoffs provided by the DM are maintained through the use of reference point technique, in other words, by assigning target values to the weight of the achievement function, a new solution can be reached that maintains the local tradeoffs. According to the PROJECT method investigated based on GRIST method and the reference point method, at the first we describe the method and then develop the PROGECT procedure.

Suppose for given positive weight vectors $w^{t}=\left\{w_{1}^{t}, \ldots, w_{k}^{t}, \ldots, w_{s}^{t}\right\}$ and $v^{t}=\left\{v_{1}^{t}, \ldots, v_{i}^{t}, \ldots, v_{m}^{t}\right\}$.

The optimal solution of the minimal model (26) is given by $\lambda^{t}=\left\{\lambda_{11}^{t}, \ldots, \lambda_{j r}^{t}, \ldots, \lambda_{n n}^{t}\right\}$, which must be an efficient solution. The optimal values of the dual variable of the kth and ith objective constraint $w_{k}\left(\bar{f}_{k}^{*}-\bar{f}_{k}(\lambda)=\gamma_{k}, v_{i}\left(\tilde{f}_{i}(\lambda)-\tilde{f}_{i}^{*}\right)=\tau_{i}\right.$ are given by $\beta_{k}^{t}$ and $\alpha_{i}^{t}$

$$
\text { Let } \bar{f}\left(\lambda^{t}\right)=\left[\bar{f}_{1}\left(\lambda^{t}\right), \ldots, \bar{f}_{k}\left(\lambda^{t}\right), \ldots, \bar{f}_{s}\left(\lambda^{t}\right)\right]^{T} \text { and }
$$
$f\left(\lambda^{t}\right)=\left[\widetilde{f}_{1}\left(\lambda^{t}\right), \ldots, \widetilde{f}_{i}\left(\lambda^{t}\right), \ldots, \widetilde{f}_{m}\left(\lambda^{t}\right)\right]^{T}$ represent the efficient solution in

the corresponding objective space. It is proven that the normal vectors $N^{t}$ at $\bar{f}\left(\lambda^{t}\right)$ and $M^{t}$ at $\widetilde{f}\left(\lambda^{t}\right)$ on the efficient frontier are given by

$$
N^{t}=\left[w_{1}^{t} \beta_{1}^{t}, \ldots, w_{k}^{t} \beta_{k}^{t}, \ldots, w_{s}^{t} \beta_{s}^{t}\right]^{T} M^{t}=\left[v_{1}^{t} \alpha_{1}^{t}, \ldots, v_{i}^{t} \alpha_{i}^{t}, \ldots, v_{m}^{t} \alpha_{m}^{t}\right]^{T}
$$

Suppose the implicit utility functions of the DM are denoted by $u(\bar{f}(\lambda)$ and $s(\tilde{f}(\lambda)$ for total outputs and inputs, respectively. The gradient of $u\left(\bar{f}(\lambda)\right.$ and $s\left(\tilde{f}(\lambda)\right.$ at the solutions $\bar{f}\left(\lambda^{t}\right)$ and $\tilde{f}\left(\lambda^{t}\right)$ aregiven by

$$
\nabla u^{t}=\frac{\partial u}{\partial \bar{f}\left(\lambda^{t}\right)}=\left[\frac{\partial u}{\partial \bar{f}_{1}^{t}}, \ldots, \frac{\partial u}{\partial \bar{f}_{k}^{t}}, \ldots, \frac{\partial u}{\partial \bar{f}_{s}^{t}}\right]^{T} \nabla s^{t}=\frac{\partial s}{\partial \widetilde{f}\left(\lambda^{t}\right)}=\left[\frac{\partial u}{\partial \widetilde{f}_{1}^{t}}, \ldots, \frac{\partial u}{\partial \widetilde{f}_{i}^{t}}, \ldots, \frac{\partial u}{\partial \widetilde{f}_{m}^{t}}\right]^{T}
$$

While $u(\bar{f}(\lambda)$ and $s(\tilde{f}(\lambda)$ are unknown in general, the utility gradients $\nabla u^{t}$ and $\nabla s^{t}$ may be estimated using the local preference information of the DM; for example, marginal rates of substitution (Yang et al, 2009). 
In the optimal condition, the gradient $\nabla u^{t}, \nabla s^{t}$ will be proportional to the normal vectors $\vec{N}$ and $\vec{M}$; otherwise, the gradients $\nabla u^{t}$ and $\nabla s^{t}$ can be projected on to the tangent plane of the efficient frontier, the projection, denoted by $\Delta u^{t}$ and $\Delta s^{t}$, are given by

$$
\begin{aligned}
& \Delta u^{t}=\left[\Delta \bar{f}_{1}^{t}, \ldots, \Delta \bar{f}_{k}^{t}, \ldots, \Delta \bar{f}_{s}^{t}\right]=\nabla u^{t}+\frac{\left[\left(\nabla u^{t}\right)^{T} N^{t}\right]}{\left.\left(N^{t}\right)^{T} N^{t}\right]} N^{t} \\
& \Delta s^{t}=\left[\Delta \widetilde{f}_{1}^{t}, \ldots, \Delta \widetilde{f}_{i}^{t}, \ldots, \Delta \widetilde{f}_{m}^{t}\right]=\nabla s^{t}-\frac{\left[\left(\nabla s^{t}\right)^{T} M^{t}\right]}{\left.\left(M^{t}\right)^{T} M^{t}\right]} M^{t}
\end{aligned}
$$

However, since the utility function is not known explicitly, a utility gradient needs to be estimated by marginal rates of substitution, $G$ and $\mathrm{R}$, which may be provided by the DM, without loss of generality, set the first objectives $\bar{f}_{1}$ and $\widetilde{f}_{1}$ as the reference objectives, then the indifference tradeoffs $G_{l k}^{t}$ and $R_{i i}^{t}$ between the first and kth and ith objectives, respectively, can be calculated using the following equations:

$$
d \bar{f}_{k}^{t}=-d \bar{f}_{1}^{t} \frac{w_{1}^{t} \beta_{1}^{t}}{w_{k}^{t} \beta_{k}^{t}}, d \tilde{f}_{i}^{t}=-d \tilde{f}_{1}^{t} \frac{v_{1}^{t} \alpha_{1}^{t}}{v_{i}^{t} \alpha_{i}^{t}}
$$

If the MPS is not achieved, then the projection $\Delta u^{t}$ and $\Delta s^{t}$ can be calculated using $G^{t}$ and $R^{t}$ as follows, denoted by $\Delta u^{\prime t}$ and $\Delta s^{\prime \prime}$, which provides a new tradeoff direction to improve the DM's utility:

$$
\begin{aligned}
& \Delta \bar{u}^{t}=\left[\Delta \bar{f}_{1}^{\prime t}, \ldots, \Delta \bar{f}_{k}^{\prime t}, \ldots, \Delta \bar{f}_{s}^{\prime t}\right]=-G^{t}+\frac{\left[\left(G^{t}\right)^{T} N^{t}\right]}{\left[\left(N^{t}\right)^{T} N^{t}\right]} N^{t} \\
& \Delta s^{t}=\left[\Delta \bar{f}_{1}^{\prime \prime t}, \ldots, \Delta \bar{f}_{i}^{\prime \prime t}, \ldots, \Delta \bar{f}_{m}^{\prime \prime t}\right]=R^{t}-\frac{\left[\left(R^{t}\right)^{T} M^{t}\right]}{\left[\left(M^{t}\right)^{T} M^{t}\right]} M^{t}
\end{aligned}
$$

Suppose $\bar{\gamma}, \bar{\tau}$ are tradeoff steps. Update the weighting parameters $v_{i}, v_{i}$ as follows:

$$
\begin{aligned}
& w_{k}^{t+1}=\frac{\bar{f}_{1}\left(\lambda^{t}\right)+\bar{\gamma} \Delta \bar{f}_{1}^{t}-\bar{f}_{1}\left(\lambda^{*}\right)}{\bar{f}_{k}\left(\lambda^{t}\right)+\bar{\gamma} \Delta \bar{f}_{k}^{\prime t}-\bar{f}_{k}\left(\lambda^{*}\right)} \quad k=1, \ldots, s \\
& v_{i}^{t+1}=\frac{\tilde{f}_{1}\left(\lambda^{t}\right)+\bar{\tau} \Delta f_{1}^{\prime \prime \prime}-\widetilde{f}_{1}\left(\lambda^{*}\right)}{\widetilde{f}_{i}\left(\lambda^{t}\right)+\bar{\tau} \Delta f_{i}^{\prime \prime t}-\widetilde{f}_{i}\left(\lambda^{*}\right)} \quad i=1, \ldots, m
\end{aligned}
$$

Replacing $w_{k}, v_{i}$ by, $w_{k}^{t+1}, v_{i}^{t+1}$ in formulation (26) and solving the model again yields a new solution $\lambda^{t+1}$ which should have a better utility than $\lambda^{t}$ for a sufficiently small $\bar{\gamma}, \bar{\tau}$, or $u\left(\lambda^{t+1}\right)>u\left(\lambda^{t}\right), s\left(\lambda^{t+1}\right)<s\left(\lambda^{t}\right)$. In the following, we will design an interactive procedure to use the above results to support the DM in searching for the MPS, summarized as follows:

\section{Steps of the Algorithm}

The ideal values of the total composite outputs and total inputs of the observed DMU using model (18) are calculated, and denoted by $f_{k}^{\prime}=\bar{f}_{k}\left(\lambda^{*}\right), f_{i}^{\prime \prime}=\tilde{f}_{i}\left(\lambda^{*}\right)$

Such that $\bar{f}\left(\lambda^{t}\right)=\left[\bar{f}_{1}\left(\lambda^{t}\right), \ldots, \bar{f}_{k}\left(\lambda^{t}\right), \ldots, \bar{f}_{s}\left(\lambda^{t}\right)\right]^{T} \quad$ and $f\left(\lambda^{t}\right)=\left[\widetilde{f}_{1}\left(\lambda^{t}\right), \ldots, \widetilde{f}_{i}\left(\lambda^{t}\right), \ldots, \widetilde{f}_{m}\left(\lambda^{t}\right)\right]^{T}$

Iteration $t=0$ Select an initial point $\lambda^{0}=\left[\lambda_{1}^{0}, \ldots, \lambda_{m}^{0}\right]$. An initial solution can be obtained by solving problem (26) by setting $w_{k}^{t}=\left(\frac{1}{f_{k}^{\prime}-Y_{k}^{\prime *}}\right) \quad \forall k=1, \ldots, s . \quad \quad v_{i}^{t}=\left(\frac{1}{X_{i}^{*}-f_{i}^{\prime \prime}}\right) \quad \forall i=1, \ldots, m$.

Also, the initial dual variable values $\beta^{t}=\left[\beta_{1}^{t}, \ldots, \beta_{s}^{t}\right]^{T}, \alpha^{t}=\left[\alpha_{1}^{t}, \ldots, \alpha_{m}^{t}\right]^{T}$ for the first $s$ and i constraint on the outputs and inputs.

Calculate the normal vectors $N^{t}$ at $\bar{f}\left(\lambda^{t}\right)$ and $M^{t}$ at $\tilde{f}\left(\lambda^{t}\right)$ using (27).

The DM is asked to obtain local indifference tradeoffs on objectives, using Eq.(30)
By solving (32) we obtain the projection of the DM's indifference tradeoffs $G^{t}$ and $R^{t}$ on to the tangent plane of the efficiency frontier and consider $\Delta u^{\prime t}=\left[\Delta f_{1}^{\prime t}, \Delta f_{2}^{\prime t}, \ldots, \Delta f_{s}^{\prime t}\right], \Delta s^{t}=\left[\Delta f_{1}^{\prime \prime t}, \Delta f_{2}^{\prime \prime}{ }^{\prime}, \ldots, \Delta f_{s}^{\prime \prime t}\right]^{T}$ , which determines the new tradeoff direction.

Calculate the desired step size $s^{t}$ could be determined by $\bar{\gamma}=\bar{\gamma}_{\max }^{t} \gamma$ and $\bar{\tau}=\bar{\tau}_{\min }^{t} \tau$ where $\bar{\gamma}_{\max }^{t}, \bar{\tau}_{\min }^{t}$ are the largest permissible step sizes and regulating factors with $0 \leq \gamma \leq 1$ and $0 \leq \alpha \leq 1$. Suppose $I^{1}$ and $J^{1}$ are the sets of the subscripts of objectives for increase and decrease, respectively. The maximum step size can be determined as follows:

$$
\begin{aligned}
& s_{\max }^{t}=\bar{\gamma}_{\max }^{t}=\max _{k \in I^{1}} \frac{f_{k}^{\prime}-\bar{f}_{k}^{t}}{\left|\Delta f_{k}^{\prime t}\right|} \\
& s_{\text {min }}^{t}=\bar{\tau}_{\text {min }}^{t}=\min _{i \in J^{1}} \frac{\widetilde{f}_{i}^{t}-f_{i}^{\prime \prime}}{\left|\Delta f_{i}^{\prime \prime}\right|}
\end{aligned}
$$

The step size is taken interval $\left[0, s_{\max }^{t}\right]$ and given that $s_{l}^{t}=\frac{l}{C_{s}} \cdot s_{\max }^{t} \quad l=1,2 \ldots, C_{S}$

The step size $s^{t}$ is taken interval $\left[s_{\min }^{t}, 0\right]$ and given that $s_{l}^{t}=\frac{l}{C_{s}} \cdot s_{\min }^{t} \quad l=1,2 \ldots, C_{S}$.

Where Cs is an integer which could be regulated to change accuracy of $s^{t}$, then, the following vectors of value on the objectives are shown to the DM:

$$
\left(f_{1}^{t}+s_{1}^{t} . \Delta f_{1}^{t}, \ldots, f_{s}^{t}+s_{l}^{t} . \Delta f_{s}^{t}\right) \quad l=1 \ldots C_{s}
$$

The new reference point is defined by $\bar{q}^{t}=\left(\bar{q}_{1}^{t}, \ldots, \bar{q}_{s}^{t}\right)$ and $\widetilde{q}^{t}=\left(\widetilde{q}_{1}^{t}, \ldots, \widetilde{q}_{m}^{t}\right)$ where

$$
\begin{array}{cc}
\bar{q}_{k}^{t}=\bar{f}_{k}^{t}+\bar{\gamma}^{t} \cdot \Delta f_{k}^{\prime t} & \forall k=1 \ldots S \\
\widetilde{q}_{i}^{t}=\widetilde{f}_{i}^{t}+\widetilde{\tau}^{t} \cdot \Delta f_{i}^{\prime \prime t} & \forall i=1 . . . m
\end{array}
$$

Solve problem (6), where

$$
\begin{array}{ll}
\bar{\mu}_{k}^{t}=\frac{1}{\left|\bar{q}_{k}^{t}-\bar{f}_{k}^{t}\right|} \quad \forall k=1 \ldots s \\
\tilde{\mu}_{i}^{t}=\frac{1}{\left|\widetilde{q}_{i}^{t}-\widetilde{f}_{i}^{t}\right|} \quad \forall i=1 \ldots m
\end{array}
$$

Let $\left(\lambda^{*}, f^{*}\right)$ be a solution.

Show the solution $\left(\lambda^{*}, f^{*}\right)$ to the DM.

If $u\left(f^{*}\right) \geq u\left(f^{t}\right)$, then let $\lambda^{t+1}=\lambda^{*}$ and $f^{t+1}=f^{*}$. Set $t=t+1$ and go to step 2 .

Otherwise, let

$$
\begin{aligned}
& \gamma^{t}:=\gamma_{\text {old }}{ }^{t} \cdot \min \left\{\mid 1-\frac{\left\|\bar{q}_{k}{ }^{t}-f^{\prime *}\right\|_{2}}{\left\|\bar{q}_{k}{ }^{t}-\bar{f}^{t}\right\|_{2} \mid}, \frac{1}{2}\right\} \leq \frac{1}{2} s_{\text {old }}^{t} \\
& \tau^{t}:=\tau_{\text {old }}{ }^{t} \cdot \min \left\{\mid 1-\frac{\left\|\widetilde{q}_{i}^{t}-f^{\prime \prime *}\right\|_{2}}{\left\|\widetilde{q}_{i}{ }^{t}-\widetilde{f}^{t}\right\|_{2} \mid}, \frac{1}{2}\right\} \leq \frac{1}{2} s_{\text {old }}^{t}
\end{aligned}
$$

And go to step 36 .

\section{Application Examples}

In this section, we apply the result investigated in the previous sections to conduct a case study. Firstly, the target model (15) is run to 
find the DEA efficiency scores. Since the DEA problem can be solved interactively without any prior judgments by transforming it into multiple objective linear programming (MOLP) formulation, then, the proposed interactive PROJECT method is applied to search for MPS on the efficient frontier.

The case study is to examine the performance measurement of UK retail banks using the data collected Wong and Yang (2004), as shown in Table 1. This table contains seven DMUs (retail banks), for each of which four inputs and two outputs are considered. The DMUs are comparable major banks in the UK including Abby National, Barclays, Halifax, HSBC, Lloyds TSB, NatWest and RBS (Royal Bank of Scotland). The four inputs are namely number of branches, number of ATMs, number of staff and asset size. The two outputs are customer satisfaction and total revenue. In the last row of Table 1 we have presented total input consumption and total output production.

We ran model (15) (solved by LINGO, a powerful professional software package) for obtaining the efficiency score. We also solved model (26) and got similar result to those obtained by model (15). As shown in Table 2.

In Table 3, only Barclays and NatWest are found to be inefficient within the reference set of the seven banks. As can be observed, we have solved just one mathematical programming problem for each model, while Yang et al solved $\mathrm{n}$ mathematical programming problem for their models, whereas they showed the equivalence between the CCR-dual model and the minimal model, In Table 4, we have compared between these models.

In the proposed interactive PROJECT method, the first step is to solve model (18) for total composite output and inputs to generate a payoff table for all DMUs. Table 5 displays the ranges of enable total output and input values when total composite outputs and total composite inputs are maximized and minimized, respectively. The maximum feasible value of the total composite output and the minimum feasible value of the total composite input are $f^{\prime}=\bar{f}\left(\lambda^{*}\right)=[64.19,165.98]^{T}$ and $f^{\prime \prime}=\bar{f}\left(\lambda^{t}\right)=[4.55,12.11,18.69,9.38]^{T}$.

In iteration 0 , the interactive procedure is generated by solving model (26) and (27), so that the intial decision variable $\lambda^{0}=\lambda_{71}=\ldots=\lambda_{77}^{0}=1$ and the normal vectors $N^{0}=[0.08,0.01] M^{0}=[1,1,0.45,1]$ can be found. For the first iteration suppose $\bar{f}_{1}, \bar{f}_{2}, \bar{f}_{4}$ are considered as the reference objectives. The optimal indifference tradeoff between $\bar{f}\left(\lambda^{0}\right)$ and $f_{1}, f_{2}$ ; also, between $\widetilde{f}\left(\lambda^{0}\right)$ and $f_{1}, f_{2}, f_{3}, f_{4}$; therefor, can be calculated using Eq. (31), such that $d \bar{f}^{0}=[1,8], \widetilde{f}^{0}=[1,1,2.2,1]$, Which results in the initial optimal indifference tradeoffs $(50.96,51.52) \Leftrightarrow(50.96-1,51.52+8)$ for outputs and $\quad(4.55,12.11,18.69,9.38) \Leftrightarrow(4.55+1,12.11+1,18.69-2.2,9.38+1)$ for inputs. If the $\mathrm{DM}$ is not satisfied with this initial optimal

\begin{tabular}{|c|c|c|c|c|c|c|c|}
\hline Dmu & Bank & No. of branches & No. of atms & No of staff & Asset size & Customer satisfaction & Total revenue \\
\hline 1 & Abby & 0.77 & 2.18 & 2.35 & 2.96 & 6.79 & 10.57 \\
\hline 2 & Barclay & 1.95 & 3.19 & 8.43 & 3.53 & 2.55 & 13.35 \\
\hline 3 & Halifax & 0.80 & 2.10 & 3.21 & 2.41 & 9.17 & 8.14 \\
\hline 4 & HSBC & 1.75 & 4.00 & 13.30 & 4.85 & 5.82 & 23.67 \\
\hline 5 & Lloyds TSB & 2.50 & 4.30 & 9.27 & 2.40 & 6.57 & 14.01 \\
\hline 6 & Nat West & 1.73 & 3.30 & 7.70 & 3.09 & 4.86 & 12.04 \\
\hline 7 & RBS & 0.65 & 1.73 & 2.65 & 1.34 & 7.28 & 7.36 \\
\hline Total & & 10.15 & 20.80 & 46.93 & 20.58 & 43.04 & 89.14 \\
\hline
\end{tabular}

Table 1: Original data set.

\begin{tabular}{|c|c|c|c|c|c|c|}
\hline Dmu & Composite $x_{1}$ & Input $x_{2}$ & Input $\times 3$ & input $x 4$ & Composite & Output \\
\hline 1 & 0.65 & 1.73 & 2.67 & 1.34 & 7.28 & 7.36 \\
\hline 2 & 0.65 & 1.73 & 2.67 & 1.34 & 7.28 & 7.36 \\
\hline 3 & 0.65 & 1.73 & 2.67 & 1.34 & 7.28 & 7.36 \\
\hline 4 & 0.65 & 1.73 & 2.67 & 1.34 & 7.28 & 7.36 \\
\hline 5 & 0.65 & 1.73 & 2.67 & 1.34 & 7.28 & 7.36 \\
\hline 6 & 0.65 & 1.73 & 2.67 & 1.34 & 7.28 & 7.36 \\
\hline 7 & 0.65 & 1.73 & 2.67 & 1.34 & 7.28 & 7.36 \\
\hline
\end{tabular}

Table 2: Equivalence between target model (15) and minima model (26).

\begin{tabular}{|c|c|c|c|c|c|c|c|c|c|c|c|}
\hline Dmu & Dea scores & $\Theta_{j 0}$ & $F^{\max }$ & $\Theta$ & Input x1 & Input x2 & Input x3 & Input $x 4$ & Output y1 & Output y2 & $\Theta=$ fmax $-\theta$ \\
\hline 1 & 1.000 & 1.000 & 1.000 & 0.000 & 0.77 & 2.18 & 2.35 & 2.96 & 6.79 & 10.57 & 0.000 \\
\hline 2 & 0.778 & 1.285 & 5.449 & 4.164 & 1.32 & 3.19 & 8.43 & 3.53 & 7.45 & 17.15 & 4.164 \\
\hline 3 & 1.000 & 1.000 & 1.238 & 0.238 & 0.80 & 2.10 & 3.21 & 2.41 & 9.17 & 8.14 & 0.238 \\
\hline 4 & 1.000 & 1.000 & 3.001 & 2.001 & 1.75 & 4.00 & 13.30 & 4.85 & 5.82 & 23.67 & 2.001 \\
\hline 5 & 1.000 & 1.000 & 1.989 & 0.989 & 2.50 & 4.30 & 9.27 & 2.40 & 6.57 & 14.01 & 0.989 \\
\hline 6 & 0.747 & 1.338 & 2.905 & 1.567 & 1.32 & 3.30 & 7.30 & 3.09 & 10.4 & 16.11 & 1.567 \\
\hline 7 & 1.000 & 1.000 & 1.000 & 0.000 & 0.65 & 1.73 & 2.67 & 1.34 & 7.28 & 7.36 & 0.000 \\
\hline
\end{tabular}

Table 3: Equivalence between CCR dual model and minima mode.

\begin{tabular}{|c|c|c|c|c|c|}
\hline Dmu & Composite & Input & Input & Input & Composite \\
\hline CCR dual model & 8.92 & 20.19 & 46.93 & 20.58 & 48.61 \\
\hline Target model & 4.55 & 12.11 & 18.69 & 9.38 & 50.96 \\
\hline
\end{tabular}

Table 4: Compared between CCR dual model and target model. 
Citation: Dalini MM, Noura AA (2017) PROJECT Method for Optimization of Target Model in DEA. Global J Technol Optim 8: 219. doi: 10.4172/22298711.1000219

indifference tradeoff, then a new set of indifference tradeoff may be proposed by the DM. The gradient projection calculated using Eq. (32) for searching the tradeoff direction with $\Delta f^{\prime 0}=[-0.001,0.006]$ and $f^{\prime}=\bar{f}\left(\lambda^{*}\right)=[0.007,0.007,-0.047,0.007]$, which mean that the DM prefers to improve $\bar{f}_{2}$ at the expense of $\bar{f}_{1}$ and also improve $\bar{f}_{3}$ at expense of $\bar{f}_{1}, \bar{f}_{2}, \bar{f}_{4}$. The optimal step size is computed by Eq. (34), resulting in

\begin{tabular}{|c|c|c|c|c|c|c|}
\hline Total output value & $\operatorname{Max} y_{1} \operatorname{Min} x_{1}$ & $\operatorname{Max} y_{2} \operatorname{Min} x_{2}$ & $\operatorname{Min} \mathrm{x}_{3}$ & $\operatorname{Min} x_{4}$ & Maximum values & Minimum Value \\
\hline$\sum_{j} y_{1 j}$ & 64.19 & 40.70 & 47.53 & 50.96 & 64.19 & \\
\hline$\sum_{j} y_{2 j}$ & 56.98 & 165.69 & 73.99 & 51.52 & 165.98 & \\
\hline$\sum_{j} x_{1 j}$ & 5.6 & 12.25 & 5.39 & 4.55 & & 4.55 \\
\hline$\sum_{j} x_{2 j}$ & 14.7 & 28 & 12.26 & 12.11 & & 12.11 \\
\hline$\sum_{j} x_{3 j}$ & 22.47 & 93.1 & 16.45 & 18.69 & & 16.45 \\
\hline$\sum_{j} x_{4 j}$ & 16.87 & 33.95 & 20.72 & 9.38 & & 9.38 \\
\hline
\end{tabular}

Table 5: Payoff table for all DMUs.

\begin{tabular}{|c|c|c|c|c|c|c|c|}
\hline $\mathbf{t}$ & $\bar{f}\left(\lambda^{t}\right)$ & $N^{t}$ & $d \bar{f}^{t}$ & $\Delta f^{\prime t}$ & $\bar{\gamma}$ & $\bar{q}^{t}$ & $\bar{\mu}^{t}$ \\
\hline 0 & $\left(\begin{array}{l}64.19 \\
56.98\end{array}\right)$ & $\left(\begin{array}{l}0.08 \\
0.01\end{array}\right)$ & $\left(\begin{array}{l}1 \\
8\end{array}\right)$ & $\left(\begin{array}{c}-0.001 \\
0.006\end{array}\right)$ & 1907 & $\left(\begin{array}{l}62.28 \\
68.42\end{array}\right)$ & $\left(\begin{array}{l}0.52 \\
0.08\end{array}\right)$ \\
\hline 1 & $\left(\begin{array}{c}40.74 \\
165.69\end{array}\right)$ & $\left(\begin{array}{c}1 \\
0.31\end{array}\right)$ & $\left(\begin{array}{c}1 \\
3.23\end{array}\right)$ & $\left(\begin{array}{c}-0.017 \\
0.054\end{array}\right)$ & 502 & $\left(\begin{array}{c}32.20 \\
174.22\end{array}\right)$ & $\left(\begin{array}{l}0.11 \\
0.11\end{array}\right)$ \\
\hline 2 & $\left(\begin{array}{l}47.53 \\
73.99\end{array}\right)$ & $\left(\begin{array}{c}1 \\
0.31\end{array}\right)$ & $\left(\begin{array}{c}1 \\
0.78\end{array}\right)$ & $\left(\begin{array}{c}-0.025 \\
0.034\end{array}\right)$ & 100 & $\left(\begin{array}{l}45.03 \\
77.39\end{array}\right)$ & $\left(\begin{array}{c}0.4 \\
0.29\end{array}\right)$ \\
\hline 3 & $\left(\begin{array}{l}50.96 \\
51.52\end{array}\right)$ & $\left(\begin{array}{c}1 \\
0.42\end{array}\right)$ & $\left(\begin{array}{c}1 \\
0.31\end{array}\right)$ & $\left(\begin{array}{c}-5 \times 10^{-5} \\
6 \times 10^{-5}\end{array}\right)$ & - & & 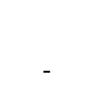 \\
\hline
\end{tabular}

Table 6: Total input for inputs 1, 2, 4 increases while the total input 3 decreases.

\begin{tabular}{|c|c|c|c|c|c|c|c|}
\hline $\mathrm{t}$ & $f^{\prime \prime t}$ & $M^{t}$ & $d \widetilde{f}^{t}$ & $\Delta f^{\prime \prime t}$ & $\bar{\tau}$ & $\widetilde{q}^{t}$ & $\tilde{\mu}^{t}$ \\
\hline 0 & $\left(\begin{array}{c}5.6 \\
14.17 \\
22.47 \\
16.87\end{array}\right)$ & $\left(\begin{array}{c}1 \\
1 \\
0.45 \\
1\end{array}\right)$ & $\left(\begin{array}{c}1 \\
1 \\
2.22 \\
1\end{array}\right)$ & $\left(\begin{array}{c}0.007 \\
0.007 \\
-0.047 \\
0.007\end{array}\right)$ & 47.66 & $\left(\begin{array}{c}5.93 \\
14.53 \\
20.22 \\
17.20\end{array}\right)$ & $\left(\begin{array}{c}3.33 \\
2.77 \\
044 \\
3.03\end{array}\right)$ \\
\hline 1 & $\left(\begin{array}{c}12.25 \\
28 \\
93.1 \\
33.95\end{array}\right)$ & $\begin{array}{c}1 \\
1 \\
0.848 \\
1\end{array}$ & $\left(\begin{array}{c}1 \\
1 \\
1.18 \\
1\end{array}\right)$ & $\left(\begin{array}{c}0.049 \\
0.049 \\
-0.167 \\
0.049\end{array}\right)$ & 21.43 & $\left(\begin{array}{c}13.30 \\
29.05 \\
89.52 \\
35\end{array}\right)$ & $\left(\begin{array}{l}0.95 \\
0.95 \\
0.28 \\
0.95\end{array}\right)$ \\
\hline 2 & $\left(\begin{array}{c}5.39 \\
12.26 \\
16.45 \\
20.72\end{array}\right)$ & $\begin{array}{c}1 \\
1 \\
0.898 \\
1\end{array}$ & $\left(\begin{array}{c}1 \\
1 \\
0.59 \\
1\end{array}\right)$ & $\left(\begin{array}{c}-0.072 \\
0.072 \\
0.187 \\
-0.980\end{array}\right)$ & 10.17 & $\left(\begin{array}{c}4.65 \\
12.99 \\
18.35 \\
10.75\end{array}\right)$ & $\left(\begin{array}{l}1.35 \\
1.36 \\
0.52 \\
0.10\end{array}\right)$ \\
\hline 3 & $\left(\begin{array}{c}4.55 \\
12.11 \\
18.69 \\
9.38\end{array}\right)$ & $\left(\begin{array}{c}1 \\
1 \\
0.97 \\
1\end{array}\right)$ & $\left(\begin{array}{c}1 \\
1 \\
0.35 \\
1\end{array}\right)$ & $\left(\begin{array}{c}-0.012 \\
0.012 \\
0.185 \\
-0.994\end{array}\right)$ & & & \\
\hline
\end{tabular}


$\bar{\gamma}_{\max }^{0}=19078, \bar{\tau}_{\min }^{0}=47.66$ to establish the step size table.

Table 6 shows that the total input for inputs 1, 2, 4 increases while the total input 3 decreases. Suppose the DM sets the target level for $\bar{f}_{3}=17.794$ and $\bar{f}_{2}=97.307$, so the regulating parameter is set to $\gamma=\tau=0.4$.

Then, in the next steps, we can calculate the new reference point using Eq. (35), and recall $\left(\bar{q}^{1}, \widetilde{q}^{1}\right)$, accordingly, with use these vectors, we can obtain the weights of minimal distance using Eq. (36), (37). Then, we go to next iteration and repeat all of the steps until the MPS are found. Tables 6 and 7 shows all stages. In this case, the computational times are also very similar for the PROJECT and GRIST methods, while it was slightly better for PROJECT.

In Conclusion, “...with use interactive PROJECT approach..." should be "... with using the interactive PROJECT approach..."

\section{Conclusion}

In this paper, we considered the equivalence relation between a target DEA model and the minimal reference point formulations, following that, with using the interactive PROJECT approach for estimating efficiency the target model in DEA, it must be pointed that any efficient solution can be generated with this interactive method, even including nonsupport efficient solutions for non-convex problems. The method based on the projection of the utility gradient onto the tangent hyper plane of the efficient set and a local search procedure of the reference point method, such that by identifying an ascending direction in the DM's utility, new values for objective are obtained. The numerical example illustrated how the equivalence model with using the interactive method can be implementing to support integrated efficiency analysis and target setting. The proposed PROJECT method has since been implemented in visual $\mathrm{C}++$ and incorporated into the software PROMOIN (interactive MOP).

\section{References}

1. Miettinen K, Makela MM (1995) Interactive bundle-based method for nondifferentiable multi-objective optimization: NIMBUS. Optimization 34: 231-246.

2. Wierzbicki AP (1980) Multiple criteria decision making theory and application. In lecture notes in Economics and Mathematical Systems 177: 468-486.

3. Yang JB (1999) Gradient projection and local region search for multiobjective optimization. Eur J Oper Res 112: 432-459.

4. Luque M, Yang J, Wong BY (2009) PROJECT method for multiobjective optimization based on gradient projection and reference points. IEEE Trans Syst Man Cybern C 39: 864-879.

5. Cooper WW (2004) Origins, uses, and relations between goal programming and data envelopment analysis. In: MCDM 2004 13: 6-11.

6. Golany B (1988) An interactive MOLP procedure for the extension of DEA to effectiveness analysis. Journal of the Operational Research Society 39: 725-734.

7. Hosseinzadeh Lotfi F, Jahanshahloo GR (2010) Relation between MOLP and DEA based on CCR dual model. Expert Syst Appl 37: 4331-4336.

8. Yang JB, Wong BY, Xu DL, Stewart TJ (2009) Integrating DEA- oriented performance assessment and target setting using interactive MOLP methods. Eur J Oper Res 195: 205-222.

9. Malekmohammadi N, Hosseinzadeh Lotfi F, Jaffar AB (2011) Target setting in data envelopment analysis using MOLP. Appl Math Model 35: 328-338.

10. Luque M, Caballero R, Molina J, Ruiz F (2007) Equivalent information for multiobjective interactive procedures. Journal of Manage. Sci 53: 125-134.

11. Charnes A, Cooper W, Rhodes E (1978) Measuring the efficiency of decision making units. Eur J Oper Res 2: 429-444.

12. Allen R, Athanassopoulos AD, Dyson RG, Thanassoulis E (1997) Weights restrictions and value judgements in data envelopment analysis. Eur $\mathrm{J}$ Oper Res 73: 13-34.

13. Wong BY, Luque M, Yang JB (2009) Using interactive multiobjective methods to solve DEA problems with value judgements. Comput Oper Res 36: 623-636.

14. Wong BY, Yang JB (2004) Data envelopment analysis and multiple criteria decision making based on the evidential reasoning approach- performance measurement of UK retail banks. Working paper, Manchester School of Management, UMIST. 